\title{
ALTERAÇÕES ELETROQUÍMICAS EM SOLOS INUNDADOS
}

\author{
ELECTROCHEMICAL CHANGES IN WETLAND SOILS
}

- REVISÃO BIBLIOGRÁFICA -

\section{Flávio Anastácio de Oliveira Camargo ${ }^{1}$ Gabriel de Araújo Santos ${ }^{2}$ Everaldo Zonta}

\section{RESUMO}

O presente trabalho tem o objetivo de revisar alguns conceitos da eletroquímica (descritos pela físico-química) dos solos inundados. As principais alterações eletroquímicas que ocorrem após a inundação são a diminuição do potencial redox, aumento do pH em solos ácidos e decréscimo em solos alcalinos, aumento da condutividade elétrica e de reações de troca iônica. Essas modificações produzidas no sistema influenciam diretamente o desenvolvimento de plantas através do controle da disponibilidade e toxidez de nutrientes, regulando a absorção na rizosfera.

Palavras-chave: atividade de elétrons, atividade de prótons, potencial redox, pe, $\mathrm{pH}$, íons, solos alagados.

\section{SUMMARY}

This paper aims to review some concepts on electrochemistry of wetland soils. The main electrochemical changes after inundation are a decrease in redox potential, an increase in $\mathrm{pH}$ in acid and a decrease in alkaline soils and increases in conductivity and ion exchange reactions. These modifications in the system might influence plant growth, by affecting the availability on toxicity of nutrients, regulating uptake in the rhizosfere.

Key words: electron activity, proton activity, redox potential, pe, $p H$, ions, wetland soils.

\section{INTRODUÇÃO}

Os estudos referentes à físico-química dos solos inundados podem ser realizados do ponto de vista das alterações eletroquímicas, que podem ocorrer nestes solos logo após a inundação. Entre as alterações observadas, as mais pronunciadas verificam-se sobre o sistema oxi-redutor, no qual o potencial de oxi-redução $\left(\mathrm{E}_{\mathrm{H}}\right)$ apresenta-se como o indicador mais importante do estado de oxidação ou redução destes solos, refletindo a posição de equilíbrio dinâmico ("steady state") existente entre os vários sistemas redox (oxigênio, ferro, manganês, nitrogênio, enxofre e carbono) e determinando a direção das reações destes sistemas quando fora do equilíbrio. Este potencial caracteriza-se através de uma ampla faixa de variações, as mudanças químicas ocorridas no solo e os efeitos sobre o desenvolvimento de cultivos em ambientes anóxicos.

Associados às alterações do $\mathrm{E}_{\mathrm{H}}$, verificam-se aumentos no $\mathrm{pH}$ de solos ácidos devido à sua redução, enquanto que em solos alcalinos, observase o decréscimo do $\mathrm{pH}$ devido à acumulação mais intensa de $\mathrm{CO}_{2}$, fazendo com que a maioria dos solos inundados se encontrem em valores próximos à neutralidade (CAMARGO et al., 1993a). Em decorrência destas condições, constata-se o aumento

\footnotetext{
${ }^{1}$ Licenciado em Ciências Agrícolas, Doutor, Professor do Departamento de Solos, Faculdade de Agronomia, Universidade Federal do Rio Grande do Sul (UFRGS), Caixa Postal 776, 90001-970, Porto Alegre, RS. Autor para correspondência.

${ }^{2}$ Engenheiro Agrônomo, PhD., Professor do Departamento de Solos, Instituto de Agronomia, Universidade Federal Rural do Rio de Janeiro.
} 
do conteúdo de eletrólitos na solução do solo, conduzindo à liberação de cátions presentes nos sítios de troca da matriz coloidal. Deste modo, alguns íons, macro e micronutrientes como o potássio, o amônio, o zinco e o cobre aumentam a sua concentração no meio e, conseqüentemente, a sua absorção pelas culturas, podendo, em alguns casos de excesso, atingir níveis de toxidez a culturas como o arroz irrigado (CAMARGO, 1992).

A complexidade aparente das transformações que ocorrem em solos inundados serão discutidas brevemente neste trabalho, através das relações entre o potencial de oxi-redução $\left(\mathrm{E}_{\mathrm{H}}\right)$ e a atividade elétrica e a de prótons sobre as características químicas dos solos após a inundação, dando-se ênfase na medição e efeito destas relações com a disponibilidade e toxidez dos elementos reduzidos as plantas cultivadas neste sistema.

\section{DESENVOLVIMENTO}

Efeitos iniciais da inundação - Um solo inundado sem perdas por infiltração, assemelha-se a um sistema fechado, isto é, não troca matéria com a periferia, mas com possibilidade de trocar energia. Quando um solo é inundado, o equilíbrio anterior é alterado, pois a água desloca o ar dos espaços porosos, criando regiões de anerobiose devido à depleção do oxigênio e o aumento de $\mathrm{CO}_{2}$, produzido pela respiração microbiana. $\mathrm{O}$ oxigênio livre presente no solo desaparece rapidamente em função da necessidade de energia (microrganismos aeróbios) para os processos biológicos, envolvendo transferência de elétrons das substâncias utilizadas como fonte de energia para as reações químicas consideradas como produto da respiração. $\mathrm{O}$ consumo de $\mathrm{O}_{2}$ deve regular a atividade de elétrons na solução do solo, de acordo com a reação:

$$
\mathrm{O}_{2}+4 \mathrm{H}^{+}+4 \mathrm{e}^{-} \mathbf{w} 2 \mathrm{H}_{2} \mathrm{O}
$$

Assim, como a liberação de $\mathrm{CO}_{2}$ irá regular a atividade de prótons na solução do solo de acordo com as reações:

$\mathrm{CO}_{2}+\mathrm{H}_{2} \mathrm{O}$ w $\mathrm{H}_{2} \mathrm{CO}_{3}{ }^{*}\left(\mathrm{CO}_{2}\right) ;\left[\mathrm{H}_{2} \mathrm{CO}_{3}{ }^{*}\right]=\mathrm{kH} P \mathrm{kO}_{2}$
$\mathrm{H}_{2} \mathrm{CO}_{3}{ }^{*}{ }^{*} \mathrm{~W} \mathrm{H}^{+}+\mathrm{HCO}_{3}{ }^{-} ; \mathrm{k}=\left(\mathrm{H}^{+}\right)\left(\mathrm{HCO}_{3}{ }^{-}\right) /\left(\mathrm{H}_{2} \mathrm{CO}_{3}{ }^{*}\right)=10^{-6,36}$ (3)

A mudança de respiração aeróbia para anaeróbia no metabolismo dos carboidratos ocorre em baixa concentração de oxigênio $\left(3.10^{-6} \mathrm{~mol} \mathrm{~L}^{-1}\right)$. Estes sistemas seguem provavelmente vias comuns até a formação do ácido pirúvico (eq. 4), utilizando compostos de carbono como substrato, componentes oxidados do solo e produtos da dissimilação da ma- téria orgânica como eletroaceptor na respiração (PONNAMPERUMA, 1972).

列 $\mathrm{O}_{6}+2 \mathrm{NAD}+2 \mathrm{ATP} 62 \mathrm{CH}_{3} \mathrm{COOH}+2 \mathrm{NADH}+2 \mathrm{H}^{+}+4 \mathrm{ATP}$

$\mathrm{Na}$ respiração aeróbia, os elétrons são captados pela nicotinamida adenina dinucleotídeo, e transferidos para o oxigênio atmosférico, através de um carreador e oxidases terminais. $\mathrm{O}$ ácido pirúvico é oxidado através do ciclo dos ácidos tricarboxílicos, onde o oxigênio é usado como eletroaceptor terminal de elétrons (eq. 5). A regeneração do $\mathrm{NAD}^{+}$permite o sistema operar ciclicamente e completar a oxidação do substrato.

$\mathrm{Na}$ ausência do oxigênio, os microrganismos anaeróbios facultativos e obrigatórios usam $\mathrm{NO}_{3}{ }^{-}, \mathrm{Mn}(\mathrm{IV}), \mathrm{Fe}(\mathrm{III}), \mathrm{SO}_{4}{ }^{=}$, etc, produtos da dissimilação da matéria orgânica $\left(\mathrm{CO}_{2}, \mathrm{~N}_{2}\right)$ e até íons $\mathrm{H}^{+}$ como eletroaceptores em sua respiração (BARTLETT e JAMES, 1993). Como a taxa de difusão do oxigênio na água é mais lenta do que no ar (LUXMOORE et $\boldsymbol{a l}$., 1970), o consumo de oxigênio pelos microrganismos é mais rápido do que o suprimento por difusão, através da fase líquida nos solos inundados. Ocorre então a formação de um gradiente de concentração de oxigênio, levando à formação de duas camadas distintas: uma de oxidação e outra de redução, pois um solo submerso ou saturado, não é uniformemente desprovido do oxigênio. Desta forma, a concentração do oxigênio pode ser elevada na camada superficial, onde a difusão de oxigênio é suficiente para o consumo dos microrganismos aeróbios. A espessura desta camada oxidada depende do equilíbrio entre difusão e consumo de oxigênio, não ultrapassando em geral a alguns milímetros (KLUDZE e DeLAUNE, 1995). Abaixo desta zona, a concentração do oxigênio decresce abruptamente para praticamente zero. Nesta região reduzida, desenvolve-se, para a maioria dos cultivos adaptados a este meio, o sistema radicular, e onde ocorrem as principais transformações eletroquímicas que afetam estas culturas (OLIVEIRA $\boldsymbol{e t}$ al., 1993a). De forma concomitante, durante o processo de redução, ocorre liberação de gases como produto final da respiração microbiana. $\mathrm{CO}_{2}$ e $\mathrm{N}_{2}$ são liberados rapidamente após o alagamento, sendo $\mathrm{CO}_{2}$ e $\mathrm{H}_{2}$ compostos gasosos típicos produzidos durante a fermentação. Encontram-se ainda quantidades apreciáveis de etileno, ácido sulfídrico, ácidos orgânicos voláteis (CAMARGO, 1992) e, finalmente, metano, produzido na fase metanogênica da decomposição anaeróbica de matéria orgânica por um 
grupo específico de bactérias (WATANABE $\boldsymbol{e} \boldsymbol{t} \boldsymbol{a l}$., 1993).

\section{inundados}

Alterações eletroquímicas em solos

As alterações que acompanham a inundação ou a submergência do solo afetam significativamente a produtividade das culturas. Estas mudanças eletroquímicas servem de partida para o deslocamento do processo de redução resultante da diminuição do oxigênio, levando a alterações significativas no potencial redox, no $\mathrm{pH}$ e na condutividade elétrica. A atividade elétrica $\left(\mathrm{a}_{\mathrm{e}}\right)$ é um índice que expressa a quantidade de elétrons no sistema. De forma semelhante ao conceito de atividade de outras substâncias, esta denota a contribuição de elétrons para a energia livre parcial do sistema. Pode ser expressado como o logarítimo da atividade de elétrons (eq. 6), assim como o pH para atividade de prótons (VELLOSO et al., 1993a).

$$
\mathrm{pe}=-\log \mathrm{a}_{\mathrm{e}}
$$

Para a reação de oxi-redução:

(oxidante) + ne $\mathbf{~}$ (redutor), onde ne é o número de elétrons, (7) no equilíbrio,

$$
\mathrm{K}=\frac{\text { (redutor) }}{\text { (oxidante) }(\mathrm{e}) "-}
$$

em condições padrões, o logarítimo de $\mathrm{k}$ é definido como $\mathrm{pe}^{\mathrm{o}}$, onde $\mathrm{k}$ é a constante de equilíbrio relacionada para as trocas na energia livre padrão $\left(\Delta \mathrm{G}^{\mathrm{o}}\right)$, de acordo com a seguinte reação:

$$
\Delta \mathrm{G}^{\mathrm{o}}=-\mathrm{RT} \operatorname{lnk}=-\mathrm{nFE}^{\mathrm{o}}
$$

$\log$ :

$$
\mathrm{E}^{\mathrm{O}}=\frac{\mathrm{RT}}{\mathrm{NF}}
$$

e se $n=1$ a $25^{\circ} \mathrm{C}$ :

$$
\mathrm{E}^{\mathrm{o}}=0,059 \log \mathrm{k}=0,059 \mathrm{pe}^{\mathrm{o}}
$$

$\mathrm{Na}$ equação (9), o valor de $\Delta \mathrm{G}^{\mathrm{o}}$ é igual à diferença entre a soma das trocas de energia livre padrão para formação de produtos com a dos reagentes, como na equação:

$\Delta \mathrm{G}^{\mathrm{o}}=\mathbf{3} \Delta \mathrm{G}_{f \text { (produtos) }}^{\mathrm{o}}-\mathbf{3} \Delta \mathrm{G}_{f \text { (reagentes) }}^{\mathrm{o}}$

Um exemplo pode ser descrito, considerando a função do elétron na redução do oxigênio:

$$
\mathrm{O}_{2}+4 \mathrm{H}^{+}+4 \mathrm{e} \text { w } \mathrm{H}_{2} \mathrm{O}
$$

$$
1
$$

$$
\mathrm{k}=\frac{}{\left(\mathrm{O}_{2}\right)\left(\mathrm{H}^{+}\right)^{4}(\mathrm{e})^{4}}
$$

Substituindo o valor de $\mathrm{k}\left(10^{-20,8}\right)$ na equação (14), aplicando o logarítimo, tem-se uma relação entre pe e $\mathrm{pH}$ :

$$
\text { pe }=20,8+1 / 4 \log P_{2}-\mathrm{pH}
$$

Na Tabela 1, são apresentadas as principais meias-reações de redução que ocorrem em solos inundados, com os respectivos $\mathrm{E}^{\mathrm{o}}$ e pe, obedecendo à seqüência termodinâmica sob condições padrões $\left(25^{\circ} \mathrm{C}\right.$ a $\left.1 \mathrm{~atm}\right)$ e no equilíbrio. A energia ganha na transferência de $1 \mathrm{~mol}$ de elétrons para um oxidante pelo $\mathrm{H}_{2}$, expressa em volts, pela conversão de calorias, é o potencial redox $\left(\mathrm{E}_{\mathrm{H}}\right)$. O termo potencial de eletrodo (potencial do equilíbrio ou potencial reversível), quando referido à reação de uma meia-pilha, isto é, o potencial desta medido contra a meia-pilha padrão de hidrogênio, é designado $E_{H}$ (potencial redox). $\mathrm{O} \mathrm{E}_{\mathrm{H}}$ constitui uma medida de intensidade, expressando essencialmente a disponibilidade, em vez da quantidade de elétrons envolvidos nos sistemas redox. $\mathrm{O} \mathrm{E}_{\mathrm{H}}$ representa o parâmetro físicoquímico mais importante na caracterização do grau de oxidação ou redução de um solo submerso (OLIVEIRA et al., 1993b). $\mathrm{E}_{\mathrm{H}}$ pode ser relacionado ao pe de acordo com a seguinte reação (quando $\mathrm{n}=$ $1)$.

$$
\mathrm{Pe}=\frac{\mathrm{F}}{\text { 2,303RT }}
$$

a $25^{\circ} \mathrm{C}$

$$
\mathrm{Pe}=\frac{\mathrm{E}_{\mathrm{H}}}{--------}
$$

A relação entre $E_{H}$ e pe em função do $\mathrm{pH}$ em sistemas redox naturais e em equilíbrio (incluindo solo) é representada na Figura 1. A linha superior representa o equilíbrio entre $\mathrm{O}_{2}$ (1atm) e a água, sendo a linha inferior representada pelo equilíbrio de $\mathrm{H}_{2}$ (1 atm) com a água. A região circulada na figura representa as faixas de abrangência em solos inundados. $\mathrm{O}$ pe dos solos inundados oscila geralmente entre 10 a $-5(\mathrm{pH} \mathrm{7,0)}$. O maior valor de pe representa uma condição oxidada, onde a atividade elétrica é baixa e o potencial de oxi-redução é elevado. Quando há valores baixos ou negativos de pe, o meio caracteriza-se pelas condições de redução. Em termos de distribuição, o maior valor de pe já cons- 
tatado é um pouco maior do que $+13,0$ e o menor, próximo de -6,0. Essa amplitude de pe pode ser ainda dividida, quanto a $\mathrm{pH} 7,0$, em três partes: solos oxidados (pe>7); solos suboxidados (pe 2 a 7) e solos anóxicos (pe<2). Os solos suboxidados diferem dos óxicos por apresentarem valores de pe baixos o suficiente para que ocorra depleção de $\mathrm{O}_{2}$, porém não baixos o suficiente para a depleção dos íons $\mathrm{SO}_{4}{ }^{2-}$.

As divisões constatadas nos diagramas pe-pH permitem, além de verificar o domínio de estabilidade entre as espécies químicas, inferir sobre a sucessão microbiana após o alagamento. VELLOSO et al. (1993a) verificaram para dois solos fluminenses, típicos de várzea, valores iniciais de pe entre 8 e 9,8 e o decréscimo destes valores, a partir já da segunda semana de inundação do solo, caracterizando-se as faixas de solo oxidado, suboxidado e anóxicos, como constatado em um dos solos no qual o valor de pe foi menor que 2 .

Em uma reação de oxi-redução, oxidantes são reduzidos, aceitando elétrons para outras substâncias e redutores são oxidados, doando elétrons para outras substâncias. Desta forma, oxidantes são eletroaceptores e redutores são eletrodoadores. Para a equação geral de oxi-redução (eq. 7) no equilíbrio, existe uma relação quantitativa entre $\mathrm{E}_{\mathrm{H}}$, o eletrodoador (redutor) e o eletroaceptor (oxidante). Se o E é mensurado sobre um eletrodo padrão de hidrogênio, ele é denominado $\mathrm{E}_{\mathrm{H}}$, de acordo com a reação abaixo:

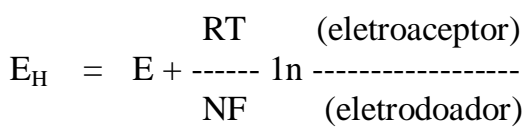

a $25^{\circ} \mathrm{C}$

$$
\mathrm{E}_{\mathrm{H}}=\mathrm{E}+\stackrel{0,059 \text { (eletroaceptor) }}{\mathrm{n}} \text { (eletrodoador) }
$$

Como o $\mathrm{E}_{\mathrm{H}}$ do sistema pode ser diretamente mensurado, esta reação é comodamente usada como um índice. Eletrodos específicos realizam esta medida onde determina-se a relação entre a substância oxidada e a reduzida, não fornecendo qualquer indicação das quantidades dessas substâncias na solução do solo. As reações químicas que envolvem trocas de elétrons influenciam os valores de $\mathrm{E}_{\mathrm{H}}$. Quando $\mathrm{E}_{\mathrm{H}}$ for positivo, define sistemas oxidados e, quando $\mathrm{E}_{\mathrm{H}}$ for negativo, sistemas reduzidos (PONNAMPERUMA, 1972). Pode-se observar na Tabela 1, o efeito da ausência do oxigênio sobre o pe

Tabela 1 - pe ${ }^{0}$ e $E^{o}$ de alguns sistemas reduzidos em solos submersos (adaptado de PONNAMPERUMA, 1972).

Sistema $\left(25^{\circ} \mathrm{C}\right) \quad \mathrm{pe}^{\mathrm{o}}(\log \mathrm{K}) \quad \mathrm{E}^{\mathrm{o}}(\mathrm{V})$

$1 / 4^{\mathrm{O}}{ }_{2}+\mathrm{H}^{+}+\mathrm{e} \mathbf{W} 1 / 2 \mathrm{H}_{2} \mathrm{O}$

20,8

$1 / 2 \mathrm{MnO}_{2}+\mathrm{H}^{+}+\mathrm{e} \mathbf{W} 1 / 2 \mathrm{Mn}^{2+}+\mathrm{H}_{2} \mathrm{O}$

20,8

$\mathrm{Fe}(\mathrm{OH})_{3}+3 \mathrm{H}^{+}+$e $\mathbf{W} \mathrm{Fe}^{2+}+3 \mathrm{H}_{2} \mathrm{O}$

17,9

1,06

$1 / 2 \mathrm{NO}_{3}{ }^{-}+\mathrm{H}^{+}+\mathrm{e}$ W $1 / 2 \mathrm{NO}_{2}^{-}+1 / 2 \mathrm{H}_{2} \mathrm{O}$

$14,1 \quad 0,83$

$1 / 8 \mathrm{SO}_{4}{ }^{2-}+5 / 4 \mathrm{H}^{+}+\mathrm{e} \mathbf{W} 1 / 8 \mathrm{H}_{2} \mathrm{~S}+1 / 2 \mathrm{H}_{2} \mathrm{O}$

5,12

0,30

$1 / 8 \mathrm{CO}_{2}+\mathrm{H}^{+}+\mathrm{e} \mathbf{W} 1 / 8 \mathrm{CH}_{4}+1 / 4 \mathrm{H}_{2} \mathrm{O}$

2,68

0,17

$\mathrm{H}^{+}+\mathrm{e} \mathbf{W} 1 / 2 \mathrm{H}_{2}$

0,00

0,00 e $\mathrm{E}_{\mathrm{H}}$, onde outros elementos químicos comportam-se como receptores de elétrons, sendo então reduzidos a partir da seqüência termodinâmica. No sistema reduzido, a matéria orgânica é o principal eletrodoador no solo (CHANG-PU e ZHI-GUANG, 1985; CAMARGO et al., 1993a), sendo que outros podem existir como no caso do $\mathrm{Fe}^{2+}, \mathrm{Mn}^{2+}, \mathrm{S}^{2-}$ e $\mathrm{H}_{2}$.

É preciso ter-se em mente, que as semireações da Tabela 1 indicam apenas que algumas reações "redox" podem ocorrer nos solos. Uma reação química favorecida por um valor elevado de logk, pode não ser favorecida cineticamente. Essas constatações aplicam-se especialmente às reações "redox", já que são extremamente lentas e de certa forma, não existe um acoplamento entre as semireações de redução e oxidação. Um exemplo pode ser descrito com a reação de oxidação da glicose acoplada à semi-reação do $\mathrm{O}_{2}$ :

Para uma solução do solo em equilíbrio

$1 / 24 \mathrm{C}_{6} \mathrm{H}_{12} \mathrm{O}_{6}+1 / 4 \mathrm{H}_{2} \mathrm{O}$ w $1 / 4 \mathrm{CO}_{2}+\mathrm{H}^{+}+\mathrm{e} \operatorname{logk}=+0,2$

$1 / 4 \mathrm{O}_{2}+\mathrm{H}^{+}+$e w $1 / 2 \mathrm{H}_{2} \mathrm{O} \quad \operatorname{logk}=+20,8$

$1 / 4 \mathrm{O}_{2}+1 / 24 \mathrm{C}_{6} \mathrm{H}_{12} \mathrm{O}_{6}$ w $1 / 4 \mathrm{CO}_{2}+1 / 4 \mathrm{H}_{2} \mathrm{O} \quad \operatorname{logk}=+21,0$

com a atmosfera $\left(P_{2}=0,21\right.$ atm $)$, o valor de $\operatorname{logk}=21,0$ prediz a oxidação completa do carbono de C (0) à C (IV). Esta predição não se verifica na solução do solo dada a persistência da matéria orgânica dissolvida nesta solução. A ausência de um acoplamento efetivo, bem como a lentidão das reações "redox" mostram a necessidade de catálise para se verificar o equilíbrio. Na solução do solo, essa catálise é promovida por microrganismos específicos 
que aceleram a semi-reação de redução ou de oxidação, produzindo valores de atividades de reagentes e produtos que concordam com as predições do equilíbrio químico. Essa possibilidade depende inteiramente do crescimento e comportamento ecológico da população microbiana e da difusão dos produtos das reações bioquímicas na solução do solo. Em alguns casos, as reações "redox" serão controladas pela dinâmica altamente variável de um sistema biológico aberto, resultando numa especiação que, quando muito corresponderá às condições locais de equilíbrio parcial. Em outros casos, incluindo os solos alagados, as reações "redox" serão controladas pelo comportamento descrito para sistemas fechados, que são efetivamente catalisados por bactérias, sendo pertinente neste caso, uma descrição de equilíbrio.

$\mathrm{Na}$ maioria dos solos, as reações de oxiredução são acompanhadas pela participação de prótons. Pela reação:

$$
\text { (oxidante) }+n \mathrm{e}^{-}+\mathrm{mH}^{+} \mathbf{w} \text { (redutor) }
$$

a $25^{\circ} \mathrm{C}$ :

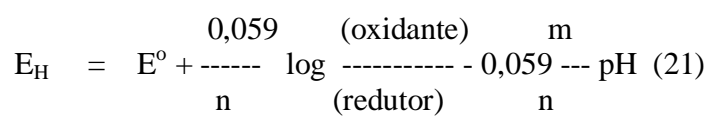

Quando a relação $\mathrm{m} / \mathrm{n}$ ou $\mathrm{H}^{+} / \mathrm{e}$ for igual a 1:

$$
\mathrm{E}_{\mathrm{H}}=\mathrm{E}^{\mathrm{o}}+\underset{\mathrm{n}}{0,059} \stackrel{\text { (oxidante) }}{\text { (redutor) }} \text {----- } \log \text {-059 } \mathrm{pH}
$$

significando que o valor teórico do coeficiente $\Delta \mathrm{E}_{\mathrm{H}} / \Delta \mathrm{pH}$ é $-59 \mathrm{mV}$ à $25^{\circ} \mathrm{C}$. A aplicação da equação (22) ao sistema inorgânico $\mathrm{O}_{2}-\mathrm{H}_{2} \mathrm{O}$ resulta em:

$$
\mathrm{E}_{\mathrm{H}}=1,23+0,0015 \log P \mathrm{O}_{2}-0,059 \mathrm{pH}
$$

e ao sistema orgânico quinona-hidroquinona a $\mathrm{pH}>8$ :

$\mathrm{O} \mathrm{pH}$, na maioria dos solos, tende à neu-

$\mathrm{E}_{\mathrm{H}}=0,699+0,0295 \log ($ quinona $) /($ hidroquinona $)-0,059 \mathrm{pH}$

tralidade após a submergência, atingindo usualmente um equilíbrio em torno de 6,5 a 7,5. A ação tamponante dos solos submersos é devido aos sistemas reduzidos do ferro, manganês e do ácido carbônico, cujas reações de oxi-redução envolvem o consumo ou a produção dos íons $\mathrm{H}^{+} / \mathrm{OH}^{-}$ (PONNAMPERUMA, 1977). Propriedades do solo, tais como conteúdo de ferro ativo e matéria orgânica, influenciam marcadamente as mudanças do $\mathrm{pH}$ (PONNAMPERUMA, 1972). CAMARGO et al. (1993a) constataram após a inundação de um solo gley pouco húmico, que recebeu diferentes doses de palha de arroz $\left(0,5,10,20\right.$ e 40t.ha $\left.{ }^{-1}\right)$, um aumento de $\mathrm{pH}$ nas primeiras 24 horas, sendo a magnitude do aumento controlada pelo nível de palha adicionada. MORAES e DYNIA (1992) observaram que a inundação provocou o aumento do $\mathrm{pH}$ da solução do solo e que este valor diminuiu após a drenagem. Em solos ácidos, o aumento do $\mathrm{pH}$, após a submergência, depende não somente da relação do íon $\mathrm{OH}^{-}$e do consumo do íon $\mathrm{H}^{+}$, mas também da relação de íons $\mathrm{H}^{+}$consumidos/elétrons consumidos. Para o aumento do pH, duas condições são necessárias: um processo de redução bem desenvolvido e a presença suficiente de ferro reduzido. A explicação provável é de que substâncias, tais como compostos ferrosos $\left(\mathrm{Fe}^{2+}\right)$, sulfeto e amônia, formadas sob condições reduzidas, são mais básicas do que quando sob condições oxidadas. O decréscimo no $\mathrm{pH}$ de solos alcalinos, após a submergência, pode ser explicado por várias alterações químicas e biológicas. A decomposição da matéria orgânica produz $\mathrm{CO}_{2}$, o qual reage com $\mathrm{H}_{2} \mathrm{O}$ para formar ácido carbônico, que se dissocia em íons $\mathrm{H}^{+}$e $\mathrm{HCO}^{3-}$. Em solos alcalinos, a diminuição do $\mathrm{pH}$ pode ser atribuída ao sistema $\mathrm{Na}_{2} \mathrm{CO}_{3}-\mathrm{H}_{2} \mathrm{O}-\mathrm{CO}_{2}$ (solos sódicos) e ao sistema $\mathrm{CaCO}_{3}-\mathrm{H}_{2} \mathrm{O}-\mathrm{CO}_{2}$ (solos calcários) (PONNAMPERUMA, 1978). O efeito do $\mathrm{pH}$ e do $\mathrm{E}_{\mathrm{H}}$ nas reações, e especialmente no equilíbrio químico final dos solos submersos, é maior quando ambos exercem este efeito, do que cada um individualmente (MANZATTO $\boldsymbol{e t} \boldsymbol{a l} .$, 1993). Essas alterações são complexas por natureza, sendo seu conhecimento indispensável ao manejo da cultura neste sistema (KLUDZE e DeLAUNE, 1995).

A condutividade elétrica (CE) é o fenômeno de transferência de eletricidade exercido pelas partículas carregadas (íons, colóides) sobre uma força aplicada em um campo elétrico. A CE dos solos inundados segue o comportamento geral da CE dos solos (JUN et al., 1985), aumentando após a submergência, atingindo um máximo e decrescendo para valores estáveis. $\mathrm{O}$ aumento na $\mathrm{CE}$, durante as primeiras semanas após o alagamento, é devido à mobilização do $\mathrm{Fe}^{2+}$ e $\mathrm{Mn}^{2+}$, acumulação de $\mathrm{NH}_{4}$, $\mathrm{HCO}_{3}$ e $\mathrm{RCOO}^{-}$e pelo deslocamento dos cátions adsorvidos nos colóides por $\mathrm{Fe}^{++}, \mathrm{Mn}^{++}$e $\mathrm{NH}_{4}^{+}$ (DeDATTA, 1983). Entretanto, alguns solos que apresentam CE elevada, a inundação pouco altera os valores iniciais (OLIVEIRA $\boldsymbol{e t}$ al., 1993a), ou verificam-se resultados inconsistentes (MORAES e DYNIA, 1992). Verifica-se também uma relação entre a CE e a força iônica que pode variar para diferentes sistemas (PONNAMPERUMA, 1977). 
A força iônica da solução é importante na determinação do coeficiente de atividade dos íons presentes, cujo conhecimento é necessário ao estudo termodinâmico do equilíbrio em solos inundados. Separando os efeitos diretos, a acumulação de íons aumenta a força iônica e altera a composição iônica da solução do solo, que afeta o equilíbrio envolvendo os nutrientes, os íons antagonistas e os íons absorvidos pelo sistema radicular. Os nutrientes adsorvidos pela argila, matéria orgânica ou oxihidróxidos de ferro, manganês e alumínio, são o nitrogênio, fósforo, enxofre, silício, boro, cobre, zinco e molibdênio (PONNAMPERUMA, 1977), sendo esta adsorção devido, possivelmente, a uma atração eletrostática ou ligação covalente.

Em função das alterações na superfície específica conduzidas pelas transformações no $\mathrm{E}_{\mathrm{H}}$, pH ou por eletrólitos, íons adsorvidos eletrostaticamente ("long range forces") ou por ligações covalentes ("short range forces"), podem ser liberados na solução do solo quando o mesmo é submerso (STUMM e MORGAN, 1981; SPOSITO, 1989). Desta maneira, a concentração do fósforo, do silício e molibdênio solúveis em água aumentam quando o solo é submerso. Este aumento na disponibilidade dos nutrientes devido à desorção dos mesmos, aumenta a disponibilidade deles para as culturas. Cobre e zinco também são liberados para a solução do solo, podendo ser fixados com o sulfeto de forma insolúvel, tal como observado por MORAES e DYNIA (1992), que constataram uma diminuição na disponibilidade de cobre com o decréscimo aparente da solubilidade após a inundação.

\section{Medida da redução do solo}

$\mathrm{O}$ estado de redução do solo pode ser definido quantitativamente através de medidas de intensidade (pe) ou de capacidade (concentração total dos produtos de redução). A determinação da concentração total dos produtos de redução é bastante ambígua e insatisfatória. Desta forma, pe constitui a medida mais usual para a determinação da capacidade de redução de um solo. Se ocorrer no solo um processo de redução no equilíbrio, pode-se utilizar a equação (7) e (8) para o cálculo de pe quando se dispor das atividades das espécies envolvidas e $\mathrm{pH}$, como mostra a equação (15), para a redução do $\mathrm{O}_{2}$ para $\mathrm{H}_{2} \mathrm{O}$. Entretanto, existem incertezas envolvidas nessa estimativa resultantes de erros nos valores de $\mathrm{pk}, \mathrm{pH}$, atividades e, principalmente, o fato de assumir a existência de um estado de equilíbrio (BARTLETT e JAMES, 1993).

Embora simples, a medida de pe na solução do solo, através de medidas de potencial de eletrodo, encontra-se sujeita a incertezas, sendo as principais referentes ao fato de que os eletrodos de platina, utilizados na determinação do potencial "redox", respondem a mais de uma semi-reação "redox". Este eletrodo pode ser freqüentemente contaminado por uma cobertura de óxidos e outras impurezas e o limite de detecção pode não ser suficiente para catar a transferência de elétrons entre as concentrações das espécies "redox", geralmente muito baixas. Um outro erro passível de ocorrer, dáse sobre o potencial de junção, cujo valor difere bastante da solução usada para aferir o eletrodo de platina (FARRELL et al., 1991).

Além destes erros intrínsecos, podem ser acrescentados erros extrínsicos, resultantes da amostragem e, em alguns casos, também da extração da solução do solo através da pressão aplicada, vácuo ou filtração. O resultado líquido dessas dificuldades é o consenso de que as medidas de $\mathrm{E}_{\mathrm{H}}$, da maioria das soluções do solo, oferecem apenas uma indicação qualitativa do pe. Estas medidas são úteis para classificar os solos em óxicos, subóxicos e anóxicos e muito pouco além disso (VELLOSO et al., 1993a).

\section{Transformações de íons}

Após a diminuição do conteúdo do $\mathrm{O}_{2}$ no solo inundado, os microrganismos anaeróbios facultativos (nitrato-redutores) reduzem o nitrato, usando-o como receptor terminal na fosforilação oxidativa da sua cadeia respiratória, conforme mostram as equações abaixo.

$\mathrm{CH}_{3} \mathrm{COOH}+\mathrm{NO}_{3} \quad 6 \quad 2 \mathrm{CO}_{2}+\mathrm{OH}^{-}+\mathrm{NH}_{3}$

$\mathrm{C}_{6} \mathrm{H}_{12} \mathrm{O}_{6}+4 \mathrm{NO}_{3}^{-} 66 \mathrm{CO}_{2}+6 \mathrm{H}_{2} \mathrm{O}+2 \mathrm{~N}_{2}$

$5 \mathrm{CH}_{3} \mathrm{COOH}+8 \mathrm{NO}_{3}^{-} 610 \mathrm{CO}_{2}+6 \mathrm{H}_{2} \mathrm{O}+80 \mathrm{H}^{-}+4 \mathrm{~N}_{2}$

A oxidação da matéria orgânica, acompanhada da conversão seqüencial do N-orgânico a Nmineral em solos inundados, pode ser esquematicamente representada conforme mostra a Figura 2. (PATRICK e REDDY, 1978). Na camada reduzida, o N-orgânico pode ser mineralizado e produzir amônio, que pode ser difundido até a camada oxidada e então ser oxidado a nitrato. Este nitrato pode sofrer nova redução (denitrificação) quando difundido em profundidade para a camada reduzida.

Com o decréscimo do $\mathrm{E}_{\mathrm{H}}$, ocorrem as reações de redução do manganês após a denitrificação. Seu uso como receptor de elétrons na respiração microbiana pela oxidação do carbono, a partir da redução dos óxidos de manganês, pode ser mostrado pela equação abaixo:

$\mathrm{CH}_{3} \mathrm{COOH}+\mathrm{MnO}_{2} \quad 62 \mathrm{CO}_{2}+\mathrm{Mn}^{2+}+4 \mathrm{H}^{+}$

Esta reação mostra que com a inundação verifica-se um aumento da concentração de manga- 
nês solúvel, podendo atingir valores tóxicos para a cultura (TADANO e YOSHIDA, 1978). Obedecendo à sequiência termodinâmica, após o Mn, os oxi-hidróxidos de $\mathrm{Fe}^{3+}$ são reduzidos a $\mathrm{Fe}^{2+}$. $\mathrm{Na}$ ausência de $\mathrm{O}_{2}, \mathrm{NO}_{3}{ }^{-}$e $\mathrm{Mn}^{4+}$, o $\mathrm{Fe}^{3+}$ pode receber elétrons da oxidação do carbono da seguinte forma:

$\mathrm{CH}_{3} \mathrm{COOH}+8 \mathrm{Fe}^{3+}+2 \mathrm{H}_{2} \mathrm{O} \quad 2 \quad 2 \mathrm{CO}_{2}+8 \mathrm{Fe}^{2+}+8 \mathrm{H}^{+}$

A coloração cinza (gley) do solo é produzida a partir da redução bacteriana do ferro em combinação com sulfetos (TANAKA e NAVASERO, 1966). O ferro sob forma reduzida $\left(\mathrm{Fe}^{2+}\right)$ aumenta após a inundação (VELLOSO et al., 1993b; MORAES e DYNIA, 1992), sendo preferencialmente absorvido pelas raízes das plantas. Em solos ácidos, a concentração do $\mathrm{Fe}^{2+}$ na solução pode também ser fitotóxico para a cultura (JUGSSUJINDA e PATRICK, 1993). A redução do sulfato, realizada por microrganismos estritamente anaeróbios, ocorre em potencial de redução muito baixo (SHU-ZHENG, 1985). A reação de oxidação do carbono e redução do sulfato podem ser esquematicamente representadas pelas equações:

$2 \mathrm{CH}_{3} \mathrm{CHOHCOOH}+\mathrm{SO}_{4}^{-} 62 \mathrm{CH}_{3} \mathrm{COOH}+2 \mathrm{H}_{2} \mathrm{O}+2 \mathrm{CO}_{2}+\mathrm{S}^{2-}$ $4 \mathrm{H}_{2}+\mathrm{SO}_{4}{ }^{2-} 6 \mathrm{~S}_{2}+4 \mathrm{H}_{2} \mathrm{O}$

O fósforo ocorre no solo sob duas formas: a orgânica, de liberação lenta, e a inorgânica, constituída de fosfatos solúveis em solução redutora (MACHADO, 1985). Sob condições de inundação, os teores de fósforo disponível aumentam, principalmente nas primeiras semanas, devendo-se isso

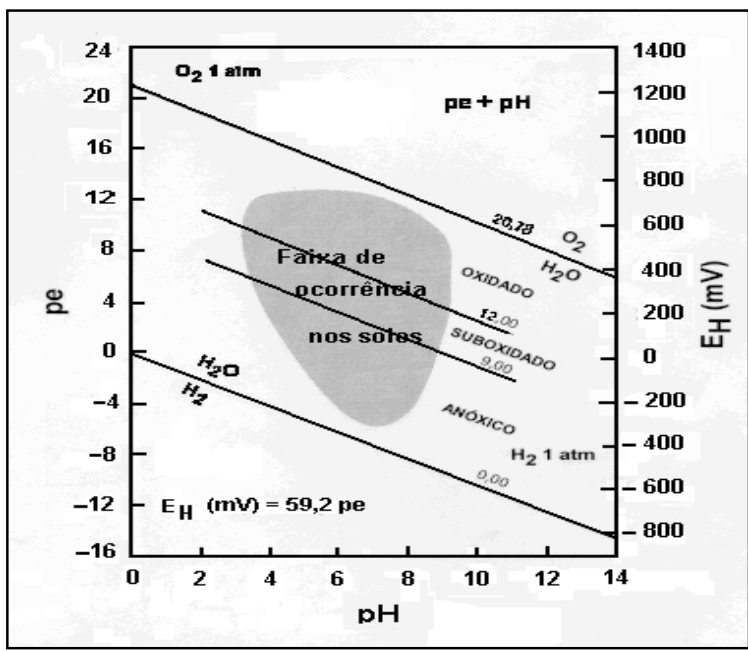

Figura 1 - Relação entre pe e $\mathrm{E}_{\mathrm{H}}$ quando no equilíbrio de sistemas "redox" em água (incluindo solo) $\left(25^{\circ} \mathrm{C}\right)$. Adaptado de Tian-Yen (1985) aos seguintes fatores: a) hidrólise dos fosfatos de ferro e de alumínio, b) liberação do fósforo adsorvido por intercâmbio aniônico nas argilas ou nos hidróxidos de $\mathrm{Fe}^{3+}$ e de $\mathrm{Al}^{3+}$ e c) redução do $\mathrm{Fe}^{3+}$ a $\mathrm{Fe}^{2+}$ com liberação do fósforo adsorvido e fixado pelo $\mathrm{Fe}^{3+}$ (VELLOSO et al., 1993b). O aumento da solubilidade do fósforo decorrente da inundação, determina uma melhor eficiência da utilização deste elemento, minimizado a dependência de fertilizantes fosfatados (GRANDE $\boldsymbol{e t}$ al., 1986). A solubilidade do silício aumenta com o tempo de inundação, promovendo a absorção mais efetiva deste elemento e, conseqüentemente, o desenvolvimento estrutural dos aerênquimas. Deste modo, favorece o aumento do suprimento de oxigênio na raiz, promovendo a "energia de oxidação", importante para a rizosfera oxidada na defesa contra a toxidez dos elementos na forma reduzida (PONNAMPERUMA, 1972).

Ao contrário dos demais elementos, o zinco decresce sua concentração após o alagamento, podendo ser devido à precipitação do $\mathrm{Zn}(\mathrm{OH})_{2}$ como resultado do aumento do $\mathrm{pH}$, precipitação do $\mathrm{ZnCO}_{3}$ devido à acumulação do $\mathrm{CO}_{2}$, resultado da decomposição da matéria orgânica e precipitação do $\mathrm{ZnS}$ sob condições altamente reduzidas. DYNIA e BARBOSA FILHO (1993) constataram que a adição de palha de arroz reduziu ligeiramente os teores de $\mathrm{Zn}$ e $\mathrm{Cu}$ extraíveis. O aumento da concentração dos cátions $\mathrm{Mn}^{2+}$ e $\mathrm{Fe}^{2+}$ na solução do solo faz-se refletir no equilíbrio complexo de troca/solução, com o deslocamento de outros cátions adsorvidos para a solução do solo. Após o alagamento, verifica-se o aumento dos teores destes cátions na solução do solo, promovendo uma maior disponibilidade (CAPECHE, 1991.

\section{Presença de plantas de arroz}

$\mathrm{O}$ arroz, crescendo em um ambiente anaeróbio, deve apresentar adaptações que permitam a proteção da rizosfera ao efeito da toxidez dos elementos nas suas formas reduzidas. Um dos mecanismos de proteção desenvolvido pela planta é a ocorrência de uma rizosfera oxidada. Durante as reações da fosforilação oxidativa, o oxigênio é enzimaticamente reduzido, formando uma molécula de água nas células das plantas. $\mathrm{O}$ suprimento às raízes das plantas pode ser feito "via solo" (sistemas aeróbios) ou "via planta", através de tecidos condutores de ar, denominados aerênquimas, que representam nas raízes do arroz, 5 a $30 \%$ do total dos tecidos. Esta capacidade do arroz, em oxidar a região da rizosfera, constitui um dos mais importantes aspectos que condicionam a planta a amenizar os efeitos tóxicos dos produtos da redução (LUXMOORE $\boldsymbol{e t}$ al., 1970). 
Em decorrência do estado oxidado e em função da energia de oxidação da rizosfera, verificase a oxidação dos elementos que se encontram na camada reduzida do solo (Figura 2). O teor de matéria orgânica no solo influi diretamente na energia de oxidação, pois o aumento neste conteúdo favorece a redução pelo suprimento de elétrons nas reações (CAMARGO et $\boldsymbol{a l} .$, 1993a). Este mecanismo conduz a uma diminuição na energia de oxidação da rizosfera, deixando-à susceptível a ação da toxidez dos produtos da redução (TIAN-YEN, 1985). Estes produtos afetam significativamente as plantas na forma de desordens nutricionais. Estas podem ser causadas pelos efeitos tóxicos do ferro (PONNAMPERUMA et al., 1955), alumínio (PATRICK e REDDY, 1978), manganês (TADANO

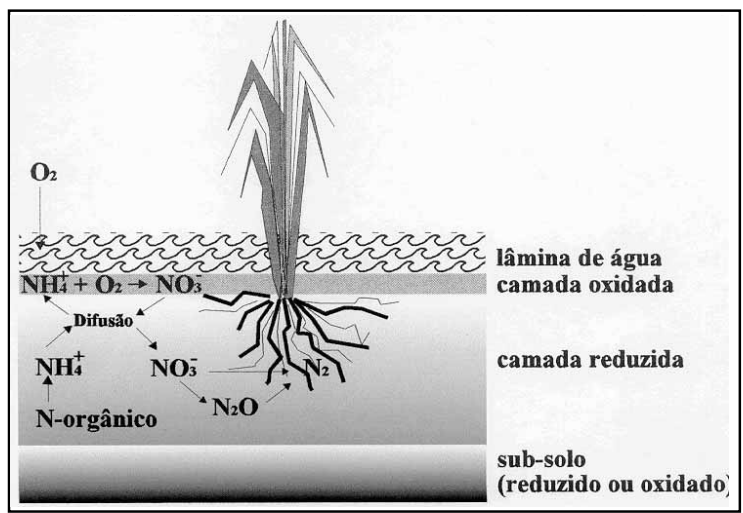

Figura 2 - Dinâmica do nitrogênio em solos inundados (adaptado de PATRICK \& READDY, 1978).

e YOSHIDA, 1978), boro (DeDATTA, 1983), sulfeto (MITSUI, 1965), ácidos orgânicos voláteis (CAMARGO et al. 1993ab) e salinidade (PATRICK e REDDY, 1978). Esquematicamente, o comportamento do sistema "redox" do solo, em função da faixa de $\mathrm{E}_{\mathrm{H}}$, tipo de reação e efeito na fisiologia das plantas podem ser sumarizados na Tabela 2.

Em geral, estes efeitos manifestam-se em função do favorecimento da redução do solo, principal- mente pela presença de matéria orgânica prontamente decomponível e do efeito do alagamento na disponibilidade destes elementos que se apresentam em concentrações elevadas na solução do solo (TIAN-YEN, 1985). Em condições anaeróbias, o problema é mais agravante, dada a baixa eficiência metabólica microbiana na conversão do carbono adicionado, levando a acumulação de compostos que afetam de forma irreversível a produtividade final, principalmente em arroz (CAMARGO et al., 1993b; CAMARGO et al., 1995ab; BRANCHER et al., 1996; BRANCHER et al., 1998).

\section{CONCLUSÕES}

As alterações das propriedades físicoquímicas que acompanham a inundação do solo são determinantes para o manejo e sustentabilidade deste ambiente. As mudanças são produzidas através de processos de redução biológica resultantes da depleção de oxigênio, com conseqüente diminuição no potencial "redox". Em solos ácidos e alcalinos, verificam-se o aumento e diminuição do $\mathrm{pH}$, respectivamente, bem como um aumento na condutividade elétrica e reações de troca iônica. Em termos de nutrição de plantas, verificam-se efeitos benéficos, proporcionados pelas alterações no sistema, tais como aumento na disponibilidade de fósforo, ferro e manganês e efeitos maléficos como a diminuição da disponibilidade de zinco, cobre, aumento excessivo de $\mathrm{Fe}^{2+}$ e formação de $\mathrm{H}_{2} \mathrm{~S}$ e ácidos orgânicos. Cultivos realizados neste sistema, como o arroz, podem ser afetados direta e indiretamente pelas mudanças através da liberação, perdas e absorção de nutrientes e formação e destruição de toxinas de origem orgânica e inorgânica.
Tabela 2 - Efeito do estado de redução do solo sobre as plantas (adaptado de TIAN-YEN, 1985).

\begin{tabular}{lclc}
\hline Estado de redução & Faixa de $\mathrm{E}_{\mathrm{H}}(\mathrm{mV})$ & \multicolumn{1}{c}{ Reações } & Crescimento de plantas \\
\hline Oxidado & $>400$ & $\begin{array}{l}\text { Predomínio de } \mathrm{O}_{2} \text { e materi- } \\
\text { ais na forma oxidada }\end{array}$ & $\begin{array}{c}\text { Benéfico para culturas } \\
\text { de sequeiro }\end{array}$ \\
Fracamente reduzido & $400-200$ & $\mathrm{O}_{2}, \mathrm{NO}_{3}{ }^{-}$e $\mathrm{Mn}^{4+}$ reduzido & $\begin{array}{c}\text { Crescimento normal do } \\
\text { arroz inundado }\end{array}$ \\
Moderadamente reduzido & $200-(-100)$ & $\begin{array}{l}\mathrm{Fe}^{3+} \text { reduzido e presença de } \\
\text { substâncias orgânicas } \\
\text { dutoras. }\end{array}$ & $\begin{array}{c}\text { Maléfico para culturas } \\
\text { re sequeiro }\end{array}$ \\
Fortemente reduzido & $<(-100)$ & $\mathrm{CO}_{2}$ e $\mathrm{H}^{+}$reduzido & $\begin{array}{c}\text { Fitotoxidez no arroz pelas } \\
\text { substâncias reduzidas }\end{array}$ \\
\hline
\end{tabular}




\section{REFERÊNCIAS BIBLIOGRÁFICAS}

BARTLETT, R.J., JAMES, B.R. Redox chemistry of soil. Advances in Agronomy, New York, v. 50, p. 151-208, 1993.

BRANCHER, A., CAMARGO, F.A.O., SANTOS, G.A Occurrence of physiological disease in flooded rice fields. Ciência Rural, Santa Maria, v. 26, n. 1, p. 149-151, 1996.

BRANCHER, A., CAMARGO, F.A.O., SANTOS, G.A. Adubação orgânica e mineral e calagem influenciando o rendimento do arroz irrigado. Pesquisa Agropecuária Brasileira, Brasília, v. 33, n. 4, p. 397-403, 1998.

CAMARGO, F.A.O. Caracterização da ação fitotóxica de ácidos orgânicos voláteis sobre a cultura do arroz. Itaguaí RS. 126p. Tese (Mestrado em Agronomia) - Curso de Pósgraduação em Agronomia, Universidade Federal Rural do Rio de Janeiro, 1992

CAMARGO, F.A.O., SANTOS, G.A., ROSSIELLO, R.O.P., $\boldsymbol{e}$ al. Produção de ácidos orgânicos voláteis pela planta de arroz sob condições anaeróbias. Revista Brasileira de Ciência do Solo, Campinas, v. 17, n. 3, p. 337-342, 1993a.

CAMARGO, F.A.O., SANTOS, G.A., ROSSIELLO, R.O.P. Efeito dos ácidos acético e butírico sobre o crescimento de plântulas de arroz. Pesquisa Agropecuária Brasileira, Brasília, v. 28, n.9, p. 1011-1018, 1993b.

CAMARGO, F.A.O., SANTOS, G.A., ROSSIELLO, R.O.P.,et al. Acúmulo de nutrientes pelo arroz influenciado pela incorporação de palha em gleissolo. Revista Brasileira de Ciência do Solo, Campinas, v. 19, n. 2, p. 243-247, 1995a.

CAMARGO, F.A.O., SANTOS, G.A., ROSSIELLO, R.O.P., et al. Incorporação de palha de arroz em um gleissolo e efeitos no rendimento da cultura do arroz irrigado. Pesquisa Agropecuária Brasileira, Brasília, v. 30, n. 7, p. 983-987, 1995b.

CAPECHE, C.L. Efeito da adubação com NPK e micronutrientes sobre a cultura do arroz irrigado em solo de várzea no Estado do Rio de Janeiro. Itaguaí - RS. 160 p. Tese (Mestrado em Agronomia) - Curso de Pós-graduação em Agronomia, Universidade Federal Rural do Rio de Janeiro, 1991.

CHANG-PU, D., ZHI-GUANG, L. Reducing substances. In: TIAN-YEN, Y. Physical chemistry of paddy soils. Berlin: Springer-Verlag, 1985. p.27-46

DeDATTA, S.K. Principles and practices of rice production. New York: John Wiley, 1981.618 p.

DYNIA, J.F., BARBOSA FILHO, M.P. Alterações de pH, Eh disponibilidade de micronutrientes para arroz irrigado em um solo de várzea tratado com calcário e palha de arroz em casa de vegetação. Revista Brasileira de Ciência do Solo, Campinas, v. 17 , n.1, p. 67-74, 1993

FARRELL, R.E., SWERHONE, G.D.W, KESSEL, C Construction and evaluation of a reference electrode assembly for use in monitoring in situ soil redox potentials Communications in Soil Science and Plant Analysis, New York, v. 22, p. 1059-1068, 1991.

GRANDE, M.A., CURI, N., QUAGGIO, J.A. Disponibilidade de fósforo pelos extratores de Mehlich e resina, em solos cultivados com arroz irrigado. Revista Brasileira de Ciência do Solo, Campinas, v. 10, p. 45-50, 1986.
JUGSUJINDA, A., PATRICK, Jr. W.H. Evaluation of toxic conditions associated with oranging symptoms of rice in a flooded Oxisol in Sumatra, Indonesia. Plant and Soil, The Hague, v. 152, p. 237-243, 1993.

JUN, W., HUI-ZHEN, S., DAO-MING, Z. Electrical conductivity. In: TIAN-YEN, Y., Physical chemistry of paddy soils. Berlin: Springer-Verlag, 1985. p. 161-177.

LUXMOORE, R.J., STOLZY, L., LETEY, J. Oxigen diffusion in the soil plant system. Agronomy Journal, Madison, v. 62, p. 317-322, 1970.

KLUDZE, H.K., DeLAUNE, R.D. Straw application on methane and oxigen exchange and growth in rice. Soil Science Society of American Journal, Madison, v. 59, p. 824-830, 1995.

MACHADO, M.O. Caracterização e adubação do solo. In EMBRAPA Fundamentos para a cultura do arroz irrigado. Campinas: Fundação Cargil, 1985. p.129-179.

MANZATTO, H.R.H., SANTOS, G.A., VELlOSO, A.C.X., et al. Influência da mistura de material de horizontes de glei pouco húmico no $\mathrm{E}_{\mathrm{H}}$ e no $\mathrm{pH}$. Revista Brasileira de Ciência do Solo, Campinas, v. 18, n. 1, p. 21-25, 1994.

MITSUI, S. Dynamic aspects of nutrient uptake. In: IRRI The mineral nutrition of the rice plant. Baltimore: Johns Hopkins Press, 1965. p. 53-62.

MORAES, J.F.V., DYNIA, J.F. Altrações nas características químicas e físico-químicas de um solo gley pouco húmico sob inundação e após drenagem. Pesquisa Agropecuária Brasileira, Brasília, v. 27, n. 2, p. 223-235, 1992.

OLIVEIRA, C., LEAL, J.R., VELLOSO, A.C.X. Processos redox em glei húmico do Estado do Rio de Janeiro: IV. Variação no "status redox" (pe+pH) de solos cultivados com arroz alagado. Revista Brasileira de Ciência do Solo, Campinas, v.17, n.1, p. 35-39, 1993a.

OLIVEIRA, C., VELLOSO, A.C.X., LEAL, J.R. Processos redox em glei húmico do Estado do Rio de Janeiro: I. Variações eletroquímicas. Revista Brasileira de Ciência do Solo, Campinas, v. 17, n. 1, p. 17-22, 1993 b.

PATRICK, W.H., REDDY, C.N. Chemical changes in rice soils In: IRRI. Soils and Rice. Los Baños:IRRI, 1978. p. 361-380.

PONNAMPERUMA, F.N. The chemistry of submerged soils. Advances in Agronomy, New York, v. 24, p. 29-96, 1972.

PONNAMPERUMA, F.N. Electrochemical changes in submerged soils and the growth of rice. In: IRRI. Soils and Rice. Los Baños:IRRI, 1978. p. 421-441.

PONNAMPERUMA, F.N., BRADFIELD, R., PEECH, M. Physiological disease of rice attributable to iron toxicity. Nature, London, v. 175, p. 265, 1955.

PONNAMPERUMA, F.N. Physicochemical properties of submerged soils in relation to fertility. Los Baños:IRRI, 1977. 32 p. Research Paper Serie, 5.

SHU-ZHENG, P. Oxigen. In: TIAN-YEN, Y., Physical chemistry of paddy soils. Berlin: Springer-Verlag, 1985. p. 47-68.

SPOSITO, G. The chemistry of soils. New York: Oxford University Press, 1989. 222p. 
STUMM, W., MORGAN, J.J. Aquatic chemistry - an introduction emphasizing chemical equilibria in natural waters. 2. ed. New York: John Wiley, 1981. 780 p.

TADANO, T., YOSHIDA, S. Chemical changes in submerged and their effect on rice growth. In: IRRI. Soils and Rice. Los Baños:IRRI, 1978. p. 399-420.

TANAKA, A., NAVASERO, S.A. Growth of the rice plant on acid sulfate soils. Soil Science and Plant Nutrition, Tokyo, v. 12 , p. $107-114,1966$.

TIAN-YEN, Y. Soil and plants. In: TIAN-YEN, Y., Physical chemistry of paddy soils. Berlin: Springer-Verlag, 1985. p. 197-214
VELLOSO, A.C.X., LEAL, J.R., OLIVEIRA, C. Processos redox em glei húmico do Estado do Rio de Janeiro: I. Avaliação do parâmetro pe+pH. Revista Brasileira de Ciência do Solo, Campinas, v. 17, n. 1, p. 23-26, 1993a.

VELlOSO, A.C.X., OLIVEIRA, C., LEAL, J.R., Processos redox em glei húmico do Estado do Rio de Janeiro: I. Variação das concentrações de Fe (II) e fosfato. Revista Brasileira de Ciência do Solo, Campinas, v. 17, n. 1, p. 27-34, 1993b.

WATANABE, A., KATOH, K., KIMURA, M. Effect of rice straw application on $\mathrm{CH}_{4}$ emission from paddy fields. I. Effect of weathering of rice straw in the field during off-crop season. Soil Science and Plant Nutrition, Tokyo, v. 39, n. 4, p. 701706, 1993.

Ciência Rural, v. 29, n. 1, 1999. 